\title{
Access to Essential Medicines in Primary Health Care Units of South Wollo Zone, Ethiopia
}

\author{
Mohammed Hussien ${ }^{*}$, Fikru Tafese ${ }^{2}$ \\ ${ }^{1}$ Department of Public Health, Wollo University, Wollo, Ethiopia \\ ${ }^{2}$ Department of Health Service Management, Jimma University, Jimma, Ethiopia \\ Email: " muhamedun@gmail.com, fikretafese@gmail.com
}

Received 2 January 2015; accepted 18 January 2015; published 22 January 2015

Copyright (C) 2015 by authors and OALib.

This work is licensed under the Creative Commons Attribution International License (CC BY). http://creativecommons.org/licenses/by/4.0/

(c) (i)

\begin{abstract}
Background: Lack of access to essential medicines is one of the most important public health problems in Ethiopia. The objective of this study was to verify availability, affordability and prices of essential medicines in primary health care units and private medicine outlets of South Wollo Zone. Methods: A facility-based cross-sectional study was undertaken in 10 primary health care units and 10 private medicine outlets. Data were collected through patient exit interview, record review, observation and interview of relevant bodies. Medicines prices were compared with international reference prices to obtain a median price ratio. The daily wage of the lowest paid government worker had been used to measure medicines affordability. Chi-square test and their p-values at the level of significance of $5 \%$ were used to define statistical associations. Odds ratios and their $95 \%$ confidence interval were used to look into the strength of association. Results: Average availability of essential medicines in primary health care units and private medicine outlets was $85.5 \%$ and $91.1 \%$ respectively. Based on prescriptions filled, $94 \%$ of the patients left the facility with all the prescribed medicines. The median price ratio of lowest price generics was 0.92 , 1.25 and 1.76 times the international reference price for public procurement price, public sector dispensing price and private sector retail price, respectively. Among the total respondents that sought treatment, $56 \%$ incurred costs of more than 12.65 Birr on medicines. Conclusion: The survey revealed efficient public procurement price, acceptable retail price and higher availability in both the public and private sectors; however medicines were less affordable in both sectors. Different financing options could improve the affordability of essential medicines for low-income population.
\end{abstract}

\section{Keywords}

Access, Availability, Affordability, Prices, Essential Medicines, South Wollo Zone

${ }^{*}$ Corresponding author. 


\section{Introduction}

Access to essential medicines is part of the fundamental right to health, and effective health services cannot be achieved without equitable universal access to essential medicines [1] [2]. Essential medicines are indispensable elements in the continuance of health care provision [2] and helpful to reduce disease burden [3]. It is estimated that by improving access to existing essential medicines, every year about 10 million lives could be saved [4] [5].

Still, lack of access to essential medicines is one of the most serious global public health problems. As much as $50 \%$ of the population in the poorest countries of Africa and Asia are denied reliable access to essential medicines [4]-[6]. Poor availability, low affordability and high medicines prices are important barriers to access to essential medicines in many low- and middle-income countries [4]-[8]. A review of data from 36 low- and middle-income countries showed that in the public sector availability ranged from $29 \%$ to $54 \%$ and private sector patients paid 9 to 25 times international reference prices (IRP) for lowest-priced generic products [9].

In Ethiopia, like in other low-income countries, poor access to essential medicines is a common public health problem. A national survey estimated that only $70 \%$ of key essential medicines were available in the public sector [10] and the cost of treatment of common diseases was found to be unaffordable to the majority of the Ethiopian people [11]. In Amhara region only 49\% of patients were able to obtain all the prescribed medicines in the health center budget pharmacies. This forces the patients to buy medications from private owned pharmacies which are almost two times more expensive than the public pharmacies. The fee waiver system also did not protect the poor from having to pay for medicines, as $44 \%$ of patients with a fee waiver were obliged to purchase medicines in the special pharmacies due to unavailability in the budget pharmacies [12].

Without concerted efforts to address high prices, unaffordable treatments and unreliable availability, lack of access to essential medicines will continue to threaten the health and well-being of people [7]. The government of Ethiopia recently reorganized the procurement and distribution of pharmaceuticals by forming a semi-autonomous institution called Pharmaceutical Fund and Supply Agency, to supply safe and affordable medicines to the public health sector on a sustainable basis [13]-[15]. The role of the insurance scheme is at its rudimentary level and the contribution to the national drug fund is only about $0.2 \%$ [13]. Currently attempts have been made by the government to introduce social health insurance for employees in the formal sectors and communitybased health insurance for the rural population [13] [14].

The objective of this study was to assess availability, affordability and prices of essential medicines in selected primary health care units (PHCUs) and private medicine outlets (PMOs) of South Wollo Zone.

\section{Materials and Methods}

A facility based, cross sectional study was conducted from February 01-30, 2012 in 10 PHCUs and 10 PMOs of South Wollo Zone, North Eastern Ethiopia. The Zone has 2 primary hospitals, 8 urban health centers and 49 rural health centers. A PHCU include primary hospital, health centers (urban and rural) and health posts. Health posts were not considered for the study, as they did not provide general outpatient care. Primary hospitals, urban health centers and rural health centers have different settings in relation to number and type of service providers, scope of services, and availability of other resources. All PHCUs in the zone were stratified to primary hospitals, urban health centers and rural health centers. Each primary hospital, urban health center and rural health center is expected to provide health services for an average population of 25,000, 40,000 and 100,000 respectively [14]. For the study, one primary hospital, two urban and seven rural health centers were selected randomly according to the proportion of the average population that each stratum is expected to serve. Based on a standardized methodology [16], the closest PMO to each of the selected PHCU was included. For PMOs not present within 10 $\mathrm{km}$ of a selected PHCU, another PMO in the urban centre was selected.

Patient sample size was calculated using single population proportion formula for descriptive cross-sectional study, assuming 5\% precision, 95\% confidence and 49\% proportion of patients who able to obtain all their prescribed medicines in public health center budget pharmacies in Amhara region [12]. Using a design effect of 2 for multistage sampling and by assuming a $10 \%$ non response rate the total sample size was 845 . The total sam- 
ple size was proportionally allocated for each of the selected PHCU based on the average daily number of patients from the outpatient department taking the last five working days prior to data collection. Finally, patients were interviewed consecutively while leaving the dispensing units.

Twenty essential medicines recommended in the national standard treatment guideline, to treat the most common health conditions in the study area were selected. These were selected from the 2010 essential medicine list of Ethiopia according to the first line therapeutic choice, at the primary health care level and have an IRP in their specified dosage form and strength.

For each selected essential medicine, data were collected based on the price and availability of Highest Price Generic (HPG) and Lowest-Price Generic (LPG) medicines using standardized data collection forms [16]. By reviewing stock cards of the listed medicines, availability on the day of data collection and the most recent price data were recorded in the PHCUs. Availability was verified by observation, and price data were obtained by asking a person in charge of the pharmacy in PMOs and for medicines which have no stock records in PHCUs. Recent public procurement prices data were obtained from one medicines wholesaler located at the center of the study area, by reviewing price records.

At PHCUs, face to face patient exit-interview was conducted and prescription papers of the interviewed patients were reviewed to obtain the relevant information, using a pre tested structured questionnaire. Nine trained data collectors consisting of three druggists and six diploma graduate nurses were employed for the data collection.

Patient interview data were double entered into EpiData version 3.1 and analyzed using SPSS version 16.0. Chi-square test and their p-values at the level of significance of $5 \%$ were used to define statistical associations. Odds Ratios (OR) and their 95\% CI were used to look into the strength of association.

Facility based data on availability and prices of medicines were double entered in to the World Health Organization/Health Action International (WHO/HAI) International Medicines Price Workbook software which has an auto-analysis feature. Based on the workbook the availability of each medicine was reported as "the percentage of stock availability of the required medicines in the facilities on the day of data collection". A medicine was recorded as "available" when any quantity of the medicine with the specified dosage form and strength was in stock in the facility at the time of data collection [16]. The following ranges have been used for describing availability [17]: <30\% very low, 30\% - 49\% low, 50\% - 80\% fairly high and $>80 \%$ high.

The median price ratio (MPR) for each medicine was calculated using the Workbook only if the medicine was available in at least four facilities. The MPR is the local median unit price of a medicine in comparison with the supplier median unit price of the IRP found in the Management Sciences for Health (MSH) international drug price indicator guide 2010 [18]. The following values for MPR were used to represent acceptable local price ratios [17]:

Procurement prices in the public sector: MPR $\leq 1$

Retail patient prices in PHCUs: MPR $\leq 1.5$

Retail patient prices in PMOs: MPR $\leq 2.5$

Affordability was estimated using median medicine prices and the average daily salary of the lowest-paid government worker and calculating the number of days' wages required to purchase seven days' course of treatment for an acute condition or a one month course of treatment for a chronic condition. The cost of dispensed medicines incurred by patients was categorized in to affordable and unaffordable based on the average daily salary of the lowest paid government worker. Treatment costs less than or equal to the average daily salary of the lowest-paid unskilled government worker was considered affordable [16]. At the time of the survey, the lowest paid government worker earned an average daily salary of 12.65 Ethiopian Birr (ETB) which equivalent to 0.732 US\$. The exchange rate of local currency to US dollars was the commercial "buy" rate (1US\$ $=17.281$ ETB) on the first day of data collection.

Ethical clearance was obtained from the Research Ethical Committee of Jimma University, School of Public Health. The purpose of the study was explained to the study participants and verbal consent was obtained from each study participant before data collection. The respondents' right to refuse or interrupting the interview at any time was respected.

\section{Results}

\subsection{Availability of Selected Essential Medicines}

Availability data was obtained in 10 PHCUs and 10 PMOs for 20 selected essential medicines. Based on se- 
lected essential medicines in stock, average availability of essential medicines was $85.5 \%$ and $86.5 \%$ and the median availability was $90 \%$ and $100 \%$ in PHCUs and PMOs, respectively. Average availability in the PMOs rises to $91.1 \%$ if Artemeter/Lumefantrine is excluded, which was exclusively available through the public sector. Average availability of essential medicines was found to be $95 \%, 80 \%$ and $85.7 \%$ in the primary hospital, urban and rural health centers, respectively (Table 1).

\subsection{Availability of Prescribed Medicines}

According to exit interview of patients who visited the PHCUs, a total of 818 patients provided a complete response to the interview with a response rate of $96.8 \%$.

On average 2.38 medicines prescribed per patient and 2.31 medicines ( $97 \%$ of the prescribed medicines) were dispensed to patients. Among the total respondents, 771 (94.3\%) left the facility with all the prescribed medicines, while $44(5.4 \%)$ received only part of and $3(0.4 \%)$ none of their prescribed medicines. Among the ten surveyed PHCUs, two rural health centers were able to provide all the prescribed medicines to their patients. In urban health centers 215 (89.6\%) of the patients received all the prescribed medicines where as in the primary hospital and rural health centers 136 (97\%) and 420 (96\%) of the patients were able to obtain all the prescribed medicines, respectively. Patients from the primary hospital were 10 times more likely to obtain the full range of

Table 1. Availability of selected essential medicines in PHCUs and PMOs of South Wollo Zone, February 2012.

\begin{tabular}{|c|c|c|}
\hline Medicines surveyed $(\mathrm{n}=20)$ & PHCUs $(n=10)$ & PMOs $(n=10)$ \\
\hline Amoxicillin & $100 \%$ & $100 \%$ \\
\hline Artemeter/Lumefantrine & $100 \%$ & $0.0 \%$ \\
\hline Chloroquine & $90 \%$ & $100 \%$ \\
\hline Chloroquine suspension & $40 \%$ & $70 \%$ \\
\hline Cimetidine & $90 \%$ & $100 \%$ \\
\hline Cloxacillin & $100 \%$ & $90 \%$ \\
\hline Co-trimoxazole & $100 \%$ & $90 \%$ \\
\hline Co-trimoxazole suspension & $90 \%$ & $100 \%$ \\
\hline Dextromethorphan & $100 \%$ & $90 \%$ \\
\hline Diclofenac & $90 \%$ & $100 \%$ \\
\hline Hydrochlorothiazide & $90 \%$ & $100 \%$ \\
\hline Magnesiumtrisilcate + Aluminiumhydroxide & $80 \%$ & $100 \%$ \\
\hline Mebendazole & $100 \%$ & $100 \%$ \\
\hline Nifedipine & $50 \%$ & $90 \%$ \\
\hline Oral Rehydration Salt & $100 \%$ & $100 \%$ \\
\hline Paracetamol & $90 \%$ & $100 \%$ \\
\hline Paracetamol suspension & $100 \%$ & $90 \%$ \\
\hline Phenobarbitone & $80 \%$ & $50 \%$ \\
\hline Salbutamol inhaler & $20 \%$ & $60 \%$ \\
\hline Tetracycline eye ointment & $100 \%$ & $100 \%$ \\
\hline Average & $85.5 \%$ & $86.5 \%$ \\
\hline
\end{tabular}

PHCU = Primary Health Care Units, PMO = Private Medicine Outlets. 
prescribed medicines compared with urban health centers $[\mathrm{OR}(95 \% \mathrm{CI})=10.0(2.8,35.9)]$ and patients from the rural health centers were approximately three and half times more likely to obtain the full range of prescribed medicines compared with urban health centers [OR $(95 \% \mathrm{CI})=3.42(1.78,6.59)]$. These variations were found to be statistically significant (p-value $<0.001$ ). For the study subjects who could not obtain the full range of prescribed medicines the reason was unavailability of medicines in the dispensaries.

Among the total respondents, 60 (7.3\%) came to the health facilities with a fee waiver, and $66(8.1 \%)$ had health insurance. Among the fee waived patients, 55 (91.7\%) received all of their medicines free of charge while the other 5 (8.3\%) receive the prescribed medicines partially. On the other hand, all patients with a health insurance were able to get the full range of prescribed medicines free of charge.

\subsection{Public Sector Procurement Prices}

The data for public procurement prices was collected from Pharmaceutical Fund and Supply Agency branch located in the center of the study area which distributes pharmaceuticals to the public health facilities. Artemeter/ Lumefantrine is charged freely to patients, hence not included in the price analysis. Public sector procurement price for both the HPG and LPG medicines was found to be 0.92 times the IRP. The MPR ranges from 0.18 to 4.10. Twelve of the 19 LPGs were procured at lower prices than the IRP; however, two medicines, Dextromethorphan and Hydrochlorothiazide were more than twice the IRP (MPR 4.1 and 3.86 respectively) (Table 2).

\subsection{Public Sector Patient Prices}

In the public sector, of the 20 essential medicines listed, 19 generics were found in 4 or more facilities. At public health facility dispensaries, patient price for 18 HPGs and LPGs was found to be 1.25 times the IRP. The prices charged to patients for the lowest priced generic medicines ranged from 0.82 to 6.26 times the IRP for Nifedipine $20 \mathrm{mg}$ and Hydrochlorothiazide $25 \mathrm{mg}$, respectively. When compared with IRP, two medicines have MPR of more than 2, while four medicines have median price below the IRP.

\subsection{Private Sector Patient Prices}

Patient prices for 19 LPGs were found to be 1.68 times the IRP in the PMOs. The prices charged to patients for the LPG medicines ranged from 0.82 times the IRP for Nifedipine $20 \mathrm{mg}$ to 6.26 times the IRP for Hydrochlorothiazide $25 \mathrm{mg}$. Both HPG and LPG equivalent products have median MPRs of 1.68. When compared with IRP, six medicines have MPR of more than 2, while one medicine has a median price below the IRP (Table 3).

\subsection{Inter-Sectoral Price Comparisons}

Primary health care units charge 35.4\% mark-ups on public procurement prices while PMOs charge $81.7 \%$ markups on public procurement prices of both HPGs and LPGs. Patient charges in the PMOs were $41.2 \%$ above patient charges in PHCUs with respect to both HPGs and LPGs.

Table 2. Summary of procurement and retail prices of essential medicines in public and private sectors of South Wollo Zone compared with IRP, February 2012.

\begin{tabular}{ccccccc}
\hline \multirow{2}{*}{ Statistics } & \multicolumn{2}{c}{ Public procurement } & \multicolumn{2}{c}{ Public retail price } & \multicolumn{2}{c}{ Private retail price } \\
\cline { 2 - 7 } & HPG & LPG & HPG & LPG & HPG & LPG \\
\hline No. of medicines & 19 & 19 & 18 & 18 & 19 & 19 \\
Median MPR & 0.92 & 0.92 & 1.25 & 1.25 & 1.68 & 1.68 \\
25\% ile MPR & 0.76 & 0.76 & 1.06 & 1.06 & 1.31 & 1.31 \\
$75 \%$ ile MPR & 1.25 & 1.13 & 1.59 & 1.59 & 2.35 & 2.26 \\
Minimum MPR & 0.18 & 0.18 & 0.82 & 0.82 & 0.82 & 0.82 \\
Maximum MPR & 4.10 & 4.10 & 6.26 & 6.26 & 6.26 & 6.26 \\
\hline
\end{tabular}

HPG = Highest Price Generics, LPG = Lowest Price Generics, MPR = Median Price Ratio. 
Table 3. Patient prices for selected medicines at PMOs of South Wollo Zone compared to IRP, February 2012.

\begin{tabular}{cc} 
Medicine name & Median MPR \\
Hydrochlorothiazide $25 \mathrm{mg}$ & 6.26 \\
Dextromethorphan syrup & 5.56 \\
Magnesiumtrisilcate + Aluminiumhydroxide tab & 2.71 \\
Diclofenac $25 \mathrm{mg}$ & 2.57 \\
Phenobarbitone $100 \mathrm{mg}$ & 2.45 \\
Mebendazole $100 \mathrm{mg}$ & 2.08 \\
Nifedipine & 0.82 \\
\hline
\end{tabular}

\subsection{Affordability Based on Selected Diseases}

Affordability of the cost of a single course of therapy for 10 conditions was measured by comparing it with the daily wage of the lowest paid government worker. On average, a lowest paid government worker would need to work for 1.3 and 1.2 days in order to purchase a course of LPG Amoxicillin from PHCUs and PMOs to treat adult pneumonia, respectively. A course of LPG co-trimoxazole suspension costs 0.6 and 0.8 days' salary of the lowest paid government worker from PHCUs and PMOs to treat child pneumonia, respectively. For the treatment of Bronchial Asthma, the lowest paid government worker would require 3.6 and 3.1 days wages to buy the LPG Salbutamol inhaler in public and private sectors respectively (Table 4).

\subsection{Affordability of Dispensed Medicines}

On exit interview of patients who visited the PHCUs, the total mean healthcare expenditure per patient was 31.5 ETB including transport cost (2.5 days wage). Medicines account $63 \%$ of the total health care expenditure. Patients spent on average 19.8 ETB (1.6 days wage) on medicines, while the median expenditure was 15 ETB. Concerning the different health facilities, patients spend on average 37.3 ETB in the primary hospital, 13 ETB in urban health centers and 18 ETB in rural health centers for medicines. The mean variation was statistically significant $(\mathrm{p}<0.01)$.

Among the 815 respondents for whom medicines were dispensed, 56\% incurred costs of more than 12.65 ETB (one days' lowest paid government salary) on medicines. For the three health facility categories 92 (65.7\%), 107 (44.6\%) and 258 (58.9\%) of patients in the primary hospital, urban health centers and rural health centers incurred costs of more than 12.65 ETB on medicines, respectively. Patients from the primary hospital were $44 \%$ less likely to get dispensed medicines with an affordable price compared with urban health centers [OR $(95 \% \mathrm{CI})=$ $0.56(0.35,0.89)]$ and patients from the rural health centers were $39 \%$ less likely to get dispensed medicines with an affordable price compared with urban health centers [OR $(95 \% \mathrm{CI})=0.61(0.43,0.86)]$. These variations were found to be statistically significant (p-value $<0.05$ ).

The number of dispensed medicines has a statistically significance association with the affordability of medicines (p-value $<0.05$ ). For a one unit increase in the number of dispensed medicines, patients were $61 \%$ less likely to get medicines with an affordable price [OR $(95 \% \mathrm{CI})=0.39(0.32,0.47)]$.

Among the total respondents for whom medicines were dispensed $(n=815), 677(83.1 \%)$ got their medicines through out of pocket expenditure while 60 (7.4\%) and 66 (8.1\%) got their medicines through fee waiver and health insurance, respectively.

\section{Discussion}

For patients to be able to access treatments adequately, medicines must be available in the public and private sectors. According to the results of this study, availability of selected essential medicines was high and on average $85.5 \%$ and $91.1 \%$ of the medicines on the survey list were available in the public health facilities and PMOs, respectively (excluding Artemeter/Lumefantrine from the private sector). This was confirmed by the finding that on average $97 \%$ of the prescribed medicines were dispensed by the public health facility pharmacies. These findings are similar with a study done in rural Amhara region in 2007, which reported average availability of $85 \%$ 
Table 4. Affordability of the cost of treatment for selected disease conditions in PHCUs and PMOs of South Wollo Zone, February 2012.

\begin{tabular}{|c|c|c|c|c|c|c|}
\hline \multirow[b]{2}{*}{ Condition } & \multirow[b]{2}{*}{ Medicine } & \multirow[b]{2}{*}{ 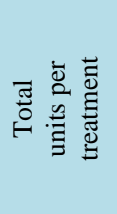 } & \multicolumn{2}{|c|}{ PHCUs } & \multicolumn{2}{|c|}{ PMOs } \\
\hline & & & 壳 芯 & 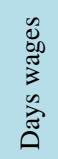 & 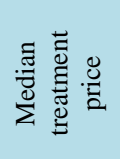 & 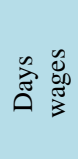 \\
\hline Asthma & Salbutamol inhaler & 200 & 52.50 & 3.6 & 39.00 & 3.1 \\
\hline Hypertension & Hydrochlorothiazide $25 \mathrm{mg}$ tab & 30 & 12.00 & 0.9 & 12.00 & 0.9 \\
\hline Hypertension & Nifedipine 20 mg, tab & 60 & 18.00 & 1.4 & 18.00 & 1.4 \\
\hline Adult ARI & Amoxicillin 500 mg, cap & 21 & 16.59 & 1.3 & 15.23 & 1.2 \\
\hline Pediatric ARI & Co-trimoxazole suspension & 100 & 7.90 & 0.6 & 10.00 & 0.8 \\
\hline Arthritis & Diclofenac $50 \mathrm{mg}$, tab & 60 & 3.38 & 0.3 & 6.00 & 0.5 \\
\hline Skin infection & Cloxacillin 500 mg, cap & 28 & 18.66 & 1.5 & 22.40 & 1.8 \\
\hline Epilepsy & Phenobarbitone $100 \mathrm{mg}$, tab & 30 & 8.42 & 0.7 & 18.00 & 1.4 \\
\hline Malaria (child) & Chloroquine suspension & 60 & 6.92 & 0.5 & 8.70 & 0.7 \\
\hline Malaria (adult) & Chloroquine $150 \mathrm{mg}$, tab & 10 & 2.10 & 0.2 & 2.75 & 0.2 \\
\hline
\end{tabular}

in public health facilities and 90\% in private pharmacies for twelve selected essential medicines [12]. Although the average availability is similar, the lists of medicines used for the survey are different. If the 10 commonly surveyed medicines in both studies are considered, public sector average availability would be $82 \%$ in the former study and $89 \%$ in the current one. Therefore, the availability of essential medicines has shown an improvement. The median availability of medicines in the public sector was $90 \%$, which is higher than $75 \%$ median availability investigated at a national level in 2004 [10]. This finding is also higher than a median availability of 65\% in Amhara region based on the 2002 national pharmaceutical sector assessment [11]. The better availability in the current study might be due to the reorganization of the pharmaceutical supply system and the presence of a single autonomous body which is responsible for the procurement and distribution of pharmaceuticals to the public health facilities.

Availability refers only the specific dosage form and strength of the medicines, but other dosage forms and strengths or alternative medicines might be available. This might undermine the availability findings.

In this survey $94 \%$ of the patients left the public health facilities with all the prescribed Medicines. This result is higher than a study in Amhara region where only $49 \%$ of the patients left the budget pharmacy with all the prescribed medicines [12]. Disparities can be due to reliance on special pharmacies to provide medicines for patients at the time of the previous study, which might be the reason for low availability in budget pharmacies. Patients seeking treatment in urban health centers were less likely to receive all prescribed medicines, compared to those treated in other facilities. In these health centers $89.6 \%$ of the patients were able to obtain all the prescribed medicines compared to $97 \%$ and $96 \%$ in the primary hospital and rural health centers respectively. This shows that $10.4 \%$ of the patients are forced to purchase medicines in private medicine outlets where medicines are relatively more expensive or forgo treatment. The variation may be due to high number of patient flow in the two urban health centers in relation to their capacity.

Availability of essential medicines could be a critical issue for voluntary insurance schemes managed by the public sector. If the members of the insurance scheme in the community are unable to get medicines from the health facilities, people will lose trust on public health facilities and may avoid being the member of the insurance scheme. In this study 66 patients were found to be the member of community based health insurance scheme, which are from one pilot district and obtain health care from three health facilities. All of these patients were able to get the full range of prescribed medicines free of charge, hence did not face the financial barriers of medicines. This can be a good lesson for program planners and decision makers to scale-up the community based health insurance scheme which is under a pilot in selected districts of the country [14].

The public sector is procuring medicines at $8 \%$ less than the published international market prices of non- 
profit generic medicine suppliers. The median MPR of procurement prices for LPGs, at 0.92 times the IRP, indicated efficient procurement. This can be compared with a previous medicine price survey in Ethiopia, in 2004 [11] which revealed a median MPR of 0.61 for procurement prices of LPGs. However, when individual medicines are considered, 7 out of 19 medicines have MPR of more than the price in the IRP indicator. This shows variability in the procurement efficiency, even with few sampled medicines.

For retail prices to patients in the PHCUs and PMOs, the median MPRs of 18 generic medicines available in both sectors were generally higher in the private sector (median MPR 1.25 and 1.76). Median MPR of below 1.5 in the public sector and below 2.5 in the private sector was considered efficient pricing. Therefore, the patient retail prices in both sectors had an acceptable median MPR. This finding in the public sector is similar to the national medicine price survey in Ethiopia in 2004 [11] which showed median MPR of 1.35. On the other hand, patient price in PMOs is cheaper than that of the 2004 national medicine price survey in Ethiopia which reported MPR of 2.25. In the current study patients charge in PMOs were only $41.2 \%$ above patient charges in public health facilities. This is different from previous studies in Ethiopia where private retail prices are much higher relative to public sector prices. A study in Amhara region in 2007 [12] showed that retail price in the PMOs was on average twice as expensive as in the public pharmacies and the 2004 national medicine price survey revealed a $67.2 \%$ difference between the two sectors. The lower PMO retail price in our study might be due to the better availability of medicines in the public health facilities, which could put pressure on private retailers to lower medicine prices to attract their customers. The median MPR of HPG and LPG products were similar at both the public health facilities and PMOs. This is because most retail outlets stocked a single generic product of a medicine, hence considered as both HPG and LPG products.

While one issue is the price of medicines, another is whether people can afford them, regardless of how cheap or expensive they are. Affordability was calculated in terms of the government worker who earns 0.732 US\$ per day, with the assumption that the income level of most of the poor is equivalent to the lowest-paid government salary per individual level. However, a substantial proportion of the population may earn less than this amount in the study area and a number of dependents may live on the wage of one person. Therefore medicines may be less affordable in the actual situation. When each disease condition was considered, medicines were less affordable in spite of their low price. This is because of low wages used in the calculation. While affordability was measured in terms of only a single medicine, it is important to note that this may not be the reality. Most conditions are treated with more medicines than calculated by this survey. Moreover, this study identified that the average number of medicines per prescription was 2.38. It is also probable that more than one family member at a time would require medicines. On the other hand, $56 \%$ of the respondents incurred a cost of more than 12.65 ETB (one days' lowest paid government salary) on medicines, which is unaffordable to the majority of the poor. Thus the calculated medicine cost represents a minute fraction of what would actually be paid by a family at any given time. Therefore, most medicines are clearly unaffordable to the majority of the people earning below, or even equivalent to, the lowest government wage.

At the time of the study, patients spent on average 19.8 ETB (1.6 days wage) on medicines. Medicines account $63 \%$ of the total direct health care expenditure (costs of transport, registration, laboratory investigation, medicines and other supplies). This result is comparable with other studies conducted in Ethiopia which reported average expenditure of 16.1 and 17.8 ETB on medicines [20] [21]. In this study, most of the patients (83.1\%) get their medicines through out of pocket expenditure. With the existence of out of pocket expenditure mechanism, most people in under developed areas may not able to afford the full cost of medicines prescribed. Therefore this financing mechanism would be a great barrier for affordability of essential medicines.

Patients from the urban health centers were more likely to get dispensed medicines with an affordable price compared with rural health centers and the primary hospital. This is because more patients from the urban health centers get dispensed medicines free of charge relative to patients from the rural health centers $(24.3 \%$ and $11.7 \%$ respectively); the higher proportion of patients with a fee waiver in urban health centers could be due to the situation that most patients without a fee waiver in urban area prefer private clinics, which is not the case for rural areas where there is few or no alternative private clinics.

In the contrary, the proportions of patients who get their medicines free of charge were nearly similar in the primary hospital and the urban health centers (22.3\% and 24.3\% respectively). The variation in the average cost of dispensed medicines may be because of the difference in the nature of disease conditions. In hospitals most medicines are prescribed to treat chronic diseases for longer period of time in comparison with health centers, which may be more expensive than medicines for acute conditions. When the number of dispensed medicines 
was increased, patients were less likely to get medicines with an affordable price. For a one unit increase in the number of dispensed medicines patients were $61 \%$ less likely to get medicines with an affordable price. This shows that irrational prescription of medicines could be an important barrier of medicines affordability.

\section{Conclusion}

In conclusion, median medicine availability was high in both the public health facilities and PMOs. Generally public sector procurement price is efficient compared with IRP. When compared with IRP, the prices of essential medicines in PHCUs and PMOs were within an acceptable range, but their prices in the private sector were relatively higher than the public sector. Even though the retail prices of essential medicines in both sectors were within an acceptable range, the costs of treatment of common disease were less affordable. Moreover the cost of prescribed medicines was not affordable for nearly half of the patients who visit the public health facilities during the study period. Therefore the affordability dimension is found to be a great barrier for reliable access to essential medicines. We recommend the Pharmaceutical Fund and Supply Agency to maintain and enhance the efficiencies of public procurement pricing, and the newly introduced health insurance schemes should be expanded and other financing options should be considered by the government to improve affordability of medicines for the low-income population. Irrational prescription of medicines has its own contribution as a barrier for medicines affordability. If health professionals are in doubt about the diagnosis of the patient's disease condition, they may prescribe more medicines to try many alternatives. This practice would increase the cost of medicines for the patient. Therefore assessing the pattern of medicine prescribing practice of health professionals is a further research area.

\section{Acknowledgements}

We would like to acknowledge Jimma University for the financial support. Our thanks also goes to South Wollo zone Health Department, Pharmaceutical Fund and Supply Agency Dessie Branch, the study health facilities, private medicine outlets, data collectors and supervisors without whom the study would not be a reality.

\section{References}

[1] Quick, J.D. (2003) Essential Medicines Twenty-Five Years on: Closing the Access Gap. Health Policy and Planning, 18, 1-3. http://dx.doi.org/10.1093/heapol/18.1.1

[2] WHO (2004) Equitable Access to Essential Medicines: A Framework for Collective Action. World Health Organization, Geneva.

[3] Tetteh, E.K. (2008) Provide Affordable Essential Medicines to African Households: The Missing Policies and Institutions for Price Containment. Social Science \& Medicine, 66, 569-581. http://dx.doi.org/10.1016/j.socscimed.2007.10.003

[4] WHO (2004) WHO Medicines Strategy 2004-2007: Countries at the Core. World Health Organization, Geneva.

[5] DFID (2004) Increasing Access to Essential Medicines in the Developing World: Government of UK Policy and Plans. Department for International Development, London.

[6] WHO (2004) The World Medicines Situation. World Health Organization, Geneva.

[7] Cameron, A., Ewen, M., Auton, M. and Abegunde, D. (2011) The World Medicines Situation 2011: Medicines Prices, Availability and Affordability. World Health Organization, Geneva.

[8] Mendis, S., Fukino, K., Cameron, A., et al. (2007) The Availability and Affordability of Selected Essential Medicines for Chronic Diseases in Six Low- and Middle-Income Countries. Bulletin of the World Health Organization, 85, 279288. http://dx.doi.org/10.2471/BLT.06.033647

[9] Cameron, A., Ewen, M., Ross-Degnan, D., Ball, D. and Laing, R. (2009) Medicine Prices, Availability, and Affordability in 36 Developing and Middle-Income Countries: A Secondary Analysis. Lancet, 373, 240-249. http://dx.doi.org/10.1016/S0140-6736(08)61762-6

[10] FMOH and WHO (2003) Assessment of the Pharmaceutical Sector in Ethiopia. Ministry of Health, Addis Ababa.

[11] FMOH and WHO (2005) Survey on Prices of Medicines in Ethiopia. Ministry of Health, Addis Ababa.

[12] Carasso, B.S., Lagarde, M., Tesfaye, A. and Palmer, N. (2009) Availability of Essential Medicines in Ethiopia: An Efficiency-Equity Trade-Off? Tropical Medicine and International Health, 14, 1394-1400.

http://dx.doi.org/10.1111/j.1365-3156.2009.02383.x 
[13] FMOH and Health Care Financing Team (2010) Ethiopia’s Fourth National Health Accounts 2007/08. Ministry of Health, Addis Ababa.

[14] FMOH (2010) Health Sector Development Program IV 2010/11-2014/15. Ministry of Health, Addis Ababa.

[15] Ryan, P. (2009) Public Sector Healthcare Supply Chain Strategic Network Analysis and Design. The World Bank, Washington DC.

[16] WHO/HAI (2008) Measuring Medicine Prices, Availability, Affordability and Price Components. World Health Organization and Health Action International, Geneva. http://www.haiweb.org/medicineprices/manual/documents.html

[17] Gelders, S., Ewen, M., Noguchi, N. and Laing, R. (2006) Price, Availability and Affordability: An International Comparison of Chronic Disease Medicines. World Health Organization, Cairo M.

[18] MSH (2010) International Drug Price Indicator Guide 2010. Management Sciences for Health, Arlington.

[19] WHO (2007) WHO Operational Package for Monitoring and Assessing Country Pharmaceutical Situations: Guide for Coordinators and Data Collectors. World Health Organization, Geneva. http://www.who.int/medicines /publications /WHO TCM_2007.2/en/ index.html

[20] Bogale, T., Haile Mariam, D. and Ali, A. (2005) Costs of Illness and Coping Strategies in a Coffee-Growing Rural District of Ethiopia. Journal of Health, Population and Nutrition, 23, 192-199.

[21] Russell, S. and Abdella, K. (2002) Too Poor to Be Sick: Coping with the Costs of Illness in East Hararghe, Ethiopia. Save the Children, London. 